\title{
ON CERTAIN SUBRINGS OF PRIME RINGS WITH DERIVATIONS
}

\author{
M. BREŠAR AND J. VUKMAN \\ (Received 19 May 1991; revised 13 August 1991) \\ Communicated by P. Schultz
}

\begin{abstract}
Let $D$ be a nonzero derivation of a noncommutative prime ring $R$, and let $U$ be the subring of $R$ generated by all $[D(x), x], x \in R$. A classical theorem of Posner asserts that $U$ is not contained in the center of $R$. Under the additional assumption that the characteristic of $R$ is not 2, we prove a more general result stating that $U$ contains a nonzero left ideal of $R$ as well as a nonzero right ideal of $R$.
\end{abstract}

1991 Mathematics subject classification (Amer. Math. Soc.): 16 W 25, 16 N 60.

Keywords and phrases: Derivation, prime ring, ideal.

Let $D$ be a nonzero derivation of a noncommutative prime ring $R$. A wellknown theorem of Posner [11] states that the subset of $R$ consisting of all $[D(x), x], x \in R$, is not contained in the center of $R$. Roughly speaking, our intention is to show that this subset is rather large; the result we shall prove is

THEOREM. Let $R$ be a noncommutative prime ring of characteristic not 2 , and let $D$ be a nonzero derivation of $R$. Then $U$, the subring of $R$ generated by all $[D(x), x], x \in R$, contains a nonzero left ideal of $R$ and a nonzero right ideal of $R$.

It is easy to see that a noncommutative prime ring cannot contain a nonzero central one-sided ideal. Thus, neglecting the fact that we have to require that the characteristic of $R$ is not 2, our result clearly generalizes

(C) 1993 Australian Mathematical Society 0263-6115/93\$A2.00+0.00 
Posner's theorem (we remark that a number of authors have already generalized Posner's theorem in several ways-see, for example, some recent papers $[1,5,12]$ where further references can be found).

On the other hand, our result is related to a theorem of Herstein [8] which asserts that if $D$ is a derivation of any ring $R$, such that $D^{3} \neq 0$, then the subring generated by all $D(x), x \in R$, contains a nonzero ideal of $R$ (see also an extension in [2]).

In order to prove the Theorem we will first prove

LEMMA 1. Let $R$ be a prime ring of characteristic not 2. If there exist nonzero derivations $D$ and $G$ of $R$ such that $G([D(x), x])=0$ for all $x \in R$, then $R$ is commutative.

This lemma, although it may appear somewhat special, is of some independent interest. Take $G$ to be an inner derivation, that is $G(x)=[a, x]$ for some $a \in R$; then we get

CoRollary. Let $R$ be a prime ring of characteristic not 2, and let $D$ be a nonzero derivation of $R$. If $a \in R$ is such that $[[D(x), x], a]=0$ for all $x \in R$ then a lies in the center of $R$.

The assumption in Posner's theorem can be written in the form

$$
[[D(x), x], y]=0 \text { for all } x, y \in R \text {. }
$$

Thus the assumption in the Corollary is much weaker. The Corollary can be compared with a result of the second named author [12] which states that a nonzero derivation $D$ of a noncommutative prime ring of characteristic not 2 cannot satisfy $[[D(x), x], x]=0$ for all $x \in R$.

Clearly, a nonzero derivation of a prime ring cannot vanish on some nonzero one-sided ideal. Therefore, Lemma 1 can be directly deduced from the Theorem. In fact, the Theorem is much more general than Lemma 1-as an illustration of this statement, note that using the Theorem it can be easily shown that Lemma 1 remains true if $G$ is a nonzero $(\alpha, \beta)$-derivation of $R$ where $\alpha$ and $\beta$ are automorphisms of $R$ (see [9; p. 170] for the notion of $(\alpha, \beta)$-derivations).

The assumption that the characteristic of $R$ is not 2 cannot be removed in Lemma 1 (and, therefore, the same is true for the Theorem). Indeed, let $R$ be any prime ring of characteristic 2 containing an element $a$ such that $a^{2}=0$; define $D$ by $D(x)=[a, x]$, and note that $D([D(x), x])=0, x \in R$.

Henceforth, $R$ will represent a prime ring with center $Z$ and extended centroid $C$. We list a few more or less well-known lemmas which will be needed in the sequel. 
LemMa 2. Suppose that the elements $a_{i}, b_{i}$ in the central closure of $R$ satisfy $\sum a_{i} y b_{i}=0$ for all $y \in R$. If $b_{i} \neq 0$ for some $i$ then the $a_{i}$ 's are C-dependent.

The explanation of the notions of the extended centroid and the central closure of a prime ring, as well as the proof of Lemma 2, can be found in [7, pp. 20-23] or [10].

A special case of Lemma 2, but very important one, is

Lemma 3. The elements $a, b$ in the central closure of $R$ are $C$-dependent if and only if $a y b=$ bya holds for all $y \in R$.

Using Lemma 2 (or even directly) one easily obtains

Lemma 4. Suppose that the elements $a, b, c$ in the central closure of $R$ satisfy $a y b=$ cya for all $y \in R$. If $a \neq 0$ then $b=c$.

Proof of Lemma 1. For the proof we need several steps.

STEP 1. For all $x, y \in R$,

$$
\begin{gathered}
{[D(x), x] G(x)+G(x)[D(x), x]=0,} \\
(D(x) x-2 x D(x)) y G(x)+G(x) y(2 D(x) x-x D(x)) \\
+D(x) y x G(x)-G(x) x y D(x)=0 .
\end{gathered}
$$

Proof. We define a mapping $B: R \times R \rightarrow R$ by

$$
B(x, y)=[D(x), y]+[D(y), x] .
$$

Linearizing $G([D(x), x])=0$ we see that $G(B(x, y))=0, x, y \in R$.

Note that

$$
B(x y, x)=B(x, x) y+x B(x, y)+D(x)[y, x] .
$$

Since $G(B(x y, x))=0, G(B(x, x))=0$, and $G(B(x, y))=0$, it follows from this identity that

$$
B(x, x) G(y)+G(x) B(x, y)+(G D)(x)[y, x]+D(x) G([y, x])=0 .
$$

In particular, if $y=x$, we have $B(x, x) G(x)+G(x) B(x, x)=0$. Since the characteristic of $R$ is not 2 this proves (1).

Replacing $y$ by $y x$ in (3), and noting that

$$
B(x, y x)=B(x, y) x+y B(x, x)+[y, x] D(x),
$$


one obtains

$$
\begin{aligned}
& B(x, x) G(y) x+B(x, x) y G(x)+G(x) B(x, y) x+G(x) y B(x, x) \\
& \quad+G(x)[y, x] D(x)+(G D)(x)[y, x] x+D(x) G([y, x]) x \\
& \quad+D(x)[y, x] G(x)=0 .
\end{aligned}
$$

According to (3) this relation reduces to

$$
B(x, x) y G(x)+G(x) y B(x, x)+G(x)[y, x] D(x)+D(x)[y, x] G(x)=0 .
$$

Transposing and collecting terms, we obtain (2).

We set $M=\{x \in R \mid D(x)$ and $G(x)$ are $C$-dependent $\}$.

STEP 2. (i) If char $R \neq 3$ then $R$ is the union of its subsets $M$ and $N=\{x \in R \mid D(x)$ and $[D(x), x]$ are $C$-dependent $\}$.

(ii) If char $R=3$ then $R$ is the union of its subsets $M$ and $\{x \in$ $R \mid[G(x), x]=0\}$.

Proof. Take $x \notin M$. We set

$$
\begin{array}{ll}
a_{1}=D(x) x-2 x D(x), & a_{4}=-G(x) x, \\
a_{2}=2 D(x) x-x D(x), & b=G(x), \\
a_{3}=x G(x), & c=D(x) .
\end{array}
$$

We have assumed that $b$ and $c$ are $C$-independent. By (2) we have

$$
a_{1} y b+b y a_{2}+c y a_{3}+a_{4} y c=0, \quad y \in R .
$$

Substituting $y c z$ for $y$ in (4) we get

$$
a_{1} y c z b+b y c z a_{2}+c y c z a_{3}+a_{4} y c z c=0 .
$$

But on the other hand we see from (4) that

$$
\left(a_{4} y c\right) z c=-a_{1} y b z c-b y a_{2} z c-c y a_{3} z c .
$$

Comparing the last two relations, we arrive at

(5) $a_{1} y(c z b-b z c)+b y\left(c z a_{2}-a_{2} z c\right)+c y\left(c z a_{3}-a_{3} z c\right)=0, \quad y, z \in R$.

By Lemma 2 there exists $z \in R$ such that $c z b-b z c \neq 0$. Therefore it follows from (5) and Lemma 2 that the elements $a_{1}, b$ and $c$ are $C$-dependent. Since $b$ and $c$ are $C$-independent we have

$$
a_{1}=\lambda b+\mu c
$$

for some $\lambda, \mu \in C$. Applying (6) in (5) we get

$$
\begin{aligned}
& b y\left(c z\left(\lambda b+a_{2}\right)-\left(\lambda b+a_{2}\right) z c\right) \\
& \quad+c y\left(c z\left(\mu b+a_{3}\right)-\left(\mu b+a_{3}\right) z c\right)=0, \quad y, z \in R .
\end{aligned}
$$


However, $b$ and $c$ are $C$-independent, therefore it follows by Lemma 2 that $c z\left(\lambda b+a_{2}\right)=\left(\lambda b+a_{2}\right) z c$ for all $z \in R$. Since $c \neq 0$, we then have $\lambda b+a_{2}=\nu c$ for some $\nu \in C$. Hence we see from (6) that $a_{1}+a_{2}=(\mu+\nu) c$; that is, $3[D(x), x]=(\mu+\nu) D(x)$. Thus, if char $R \neq 3$ this means that $x \in N$.

Now suppose that char $R=3$. Note that in this case $a_{2}=-a_{1}$. Therefore (4) and (6) yield $c y\left(\mu b+a_{3}\right)=\left(\mu b-a_{4}\right) y c, y \in R$. Hence $a_{3}=-a_{4}$ by Lemma 4. That is, $G(x) x=x G(x)$.

SteP 3. (i) If $x \in M$ then either $G(x)=0$ or $[D(x), x]=0$.

(ii) If $x \in M$ then either $D(x)=0$ or $[G(x), x]=0$.

Proof. Take $u \in M$ such that $G(u) \neq 0$. We want to show that $[D(u), u]$ $=0$. Of course we may assume that $D(u) \neq 0$. Thus $G(u)=\alpha D(u)$ for some $\alpha \neq 0$ in $C$. Observe that (2) then implies

$$
-2 \alpha u D(u) y D(u)+2 \alpha D(u) y D(u) u=0, \quad y \in R .
$$

Since $\alpha \neq 0$ and the characteristic of $R$ is not 2, it follows that $D(u) y D(u) u$ $=u D(u) y D(u), y \in R$. Consequently $D(u) u=u D(u)$ by Lemma 4. Thus (i) is proved. Analogously one proves (ii).

STEP 4. If char $R=3$ then $R$ is commutative.

Proof. From (ii) in Step 2 and (ii) in Step 3 we see that given $x \in R$, we have either $D(x)=0$ or $[G(x), x]=0$. We claim that $[G(x), x]=0$ for all $x \in R$. Suppose this does not hold for some $x \in R$. Then, of course, $D(x)=0$. Since $D \neq 0$ we have $D(y) \neq 0$ for some $y \in R$. Thus $[G(y), y]=0$. Now, consider the elements $x+y$ and $x-y$. We have $D(x+$ $y) \neq 0, D(x-y) \neq 0$, so that $[G(x+y), x+y]=0,[G(x-y), x-y]=0$; note that these two relations contradict the assumption that $[G(x), x] \neq 0$.

Thus $[G(x), x]=0$ holds for all $x \in R$. By Posner's theorem, $R$ is commutative.

We assume henceforth that $\operatorname{char} R \neq 3$. 0 .

STEP 5. If $x \in N$ then either $[D(x), x]=0$ or $D(x) G(x)+G(x) D(x)=$

Proof. Take $x \in N$. Since $D(x)=0$ implies $[D(x), x]=0$ we may assume that $D(x) \neq 0$ and it follows that $[D(x), x]=\beta D(x)$ for some $\beta \in C$. By (1) we then see that $\beta(D(x) G(x)+G(x) D(x))=0$. Thus either $\beta=0$, i.e., $[D(x), x]=0$, or $D(x) G(x)+G(x) D(x)=0$.

STEP 6. $R$ is the union of its subsets $P=\{x \in R \mid[D(x), x]=0\}$ and $Q=\{x \in R \mid D(x) G(x)+G(x) D(x)=0\}$. 
Proof. Combine (i) in Step 2, (i) in Step 3, and Step 5.

STEP 7. Either $P=R$ or $Q=R$.

Proof. We define biadditive mappings $A_{1}: R \times R \rightarrow R$ and $A_{2}: R \times R \rightarrow$ $R$ by

$$
\begin{aligned}
& A_{1}(x, y)=[D(x), y], \\
& A_{2}(x, y)=D(x) G(y)+G(x) D(y) .
\end{aligned}
$$

By Step 6 we see that for any $x \in R$, either $A_{1}(x, x)=0$ (that is, $x \in P$ ) or $A_{2}(x, x)=0$ (that is, $x \in Q$ ). Suppose that $P \neq R$ and $Q \neq R$. Thus there exist $x, y \in R$ such that $A_{1}(x, x) \neq 0$ and $A_{2}(y, y) \neq 0$. In this case we have $A_{1}(y, y)=0$ and $A_{2}(x, x)=0$.

Suppose that $x+y \in P$; that is, $A_{1}(x+y, x+y)=0$. Since $A_{1}(y, y)=0$ this relation can be written in the form

$$
A_{1}(x, x)+A_{1}(x, y)+A_{1}(y, x)=0 .
$$

If also $x-y$ lies in $P$, then it follows that $A_{1}(x, x)-A_{1}(x, y)-A_{1}(y, x)=$ 0 . But then (7) yields $A_{1}(x, x)=0$, contrary to the assumption. Thus $x-y \in Q$. That is, $A_{2}(x-y, x-y)=0$, and therefore

$$
-A_{2}(x, y)-A_{2}(y, x)+A_{2}(y, y)=0
$$

since $A_{2}(x, x)=0$. Consider the element $x+2 y$. If this element lies in $P$ then we have $A_{1}(x, x)+2 A_{1}(x, y)+2 A_{1}(y, x)=0$-but then it follows from (7) that $A_{1}(x, x)=0$. Thus $x+2 y \in Q$. Consequently $2 A_{2}(x, y)+2 A_{2}(y, x)+4 A_{2}(y, y)=0$. According to (8) we then have $6 A_{2}(y, y)=0$, which leads to $A_{2}(y, y)=0$ since we have assumed that the characteristic of $R$ is different from 2 and 3. But this also contradicts the assumption.

Thus we have proved that $x+y \notin P$, and so $x+y \in Q$. In a similar fashion as above one shows that this is impossible if $x \notin P$ and $y \notin Q$.

STEP 8. $R$ is commutative.

Proof. Suppose that $Q=R$, that is, $D(x) G(x)+G(x) D(x)=0, x \in R$. We claim that this relation contradicts the assumption that $D$ and $G$ are nonzero (this assertion is also presented in our paper [4]; however, we include the proof since it is rather short).

Note that any derivations $D$ and $G$ satisfy

$$
(D G)\left(x^{2}\right)=(D G)(x) x+D(x) G(x)+G(x) D(x)+x(D G)(x) .
$$

If $D(x) G(x)+G(x) D(x)=0, x \in R$, we then have $(D G)\left(x^{2}\right)=(D G)(x) x+$ $x(D G)(x), x \in R$. That is, $D G$ is a Jordan derivation. A theorem of Herstein then tells us that $D G$ is a derivation (see [6; Theorem 3.3] or [3] where 
a brief proof is presented). But the composition of two nonzero derivations of a prime ring of characteristic not 2 cannot be a derivation [11; Theorem 1].

Thus $Q \neq R$. By Step 7 we then have $P=R$, that is, $[D(x), x]=0, x \in$ $R$. But then Posner's theorem tells us that $R$ is commutative.

Proof of the Theorem. We assume that the Theorem is not true. By left-right symmetry we may assume that $U$ does not contain nonzero left ideals.

STEP 1. For all $u \in U,[D(u), u]=0$.

Proof. As above, by $B(x, y)$ we denote $[D(x), y]+[D(y), x]$. Since $B(x, y)=[D(x+y), x+y]-[D(x), x]-[D(y), y]$ we see that $B(x, y) \in$ $U, x, y \in R$. Expanding $B\left(x^{2}, x\right)$ we then get $B(x, x) x+x B(x, x) \in U$. Replacing $x$ by $x+u$ we arrive at

$$
\begin{aligned}
& B(x, x) u+u B(x, x)+2 B(x, u) u+2 u B(x, u) \\
& +2 B(x, u) x+2 x B(x, u)+B(u, u) x+x B(u, u) \in U
\end{aligned}
$$

If $u \in U$ then the first four summands lie in $U$, so it follows that

$$
2 B(x, u) x+2 x B(x, u)+B(u, u) x+x B(u, u) \in U
$$

for $u \in U, x \in R$. A substitution $-x$ for $x$ clearly yields

$$
2 B(u, u) x+2 x B(u, u) \in U, \quad u \in U, \quad x \in R .
$$

Hence, given $u, v \in U, x \in R$, we have that

$$
x[v, 2 B(u, u)]=\{2 B(u, u) x v+2 x v B(u, u)\}-(2 B(u, u) x+2 x B(u, u)) v
$$

lies in $U$. That is, $U$ contains a left ideal $R[v, 2 B(u, u)]$ where $u$ and $v$ are arbitrary elements in $U$. By assumption it follows that $[v, 2 B(u, u)]=$ $0, u, v \in U$. In particular, $2 B(u, u)$ commutes with all elements of the form $[D(x), x], x \in R$. Therefore $2 B(u, u) \in Z$ by the Corollary. But then (9) gives $R(4 B(u, u)) \in U$. By assumption, $4 B(u, u)=0$ and so $B(u, u)=0$; that is, $2[D(u), u]=0$.

STEP 2. For all $u \in U, x \in R,[x, u] D(u) \in U$ and $D(u)[x, u] \in U$.

Proof. Noting that $B(u x, u)=B(u, u) x+u B(x, u)+D(u)[x, u]$ and using Step 1 it follows that $D(u)[x, u] \in U$. Expanding $B(x u, u)$ one obtains that $[x, u] D(u) \in U$.

STEP 3. For every $u \in U$ there exists $\lambda(u) \in C$ so that $D(u) u=\lambda(u) D(u)$.

Proof. Take $u \in U$. For simplicity we denote $D(u)$ by $a$, and by $\delta$ we denote the inner derivation $\delta(x)=[x, u]$. By Step 1 we have $\delta(a)=0$, and 
by Step 2 we have $\delta(x) a \in U, x \in R$. Taking $x a \delta(y)$ for $x$ we then get $(\delta(x) a)(\delta(y) a)+x a \delta^{2}(y) a \in U$. The first term is in $U$, so it follows that for any $y \in R, U$ contains the left ideal $R a \delta^{2}(y) a$. Consequently $a \delta^{2}(y) a=0$, $y \in R$. Therefore, observing that $\delta^{2}(y a z)=\delta^{2}(y) a z+2 \delta(y) a \delta(z)+y a \delta^{2}(z)$ and using $a \delta^{2}(y a z) a=0, a \delta^{2}(y) a=0$ and $a \delta^{2}(z) a=0$, one obtains that $a \delta(y) a \delta(z) a=0, y, z \in R$. Replacing $y$ by $z a y$ and expanding the relation so obtained, it follows at once that $a \delta(z)$ aya $a(z) a=0, y, z \in R$. But then $a \delta(z) a=0$ by the primeness of $R$. By definitions of $a$ and $\delta$, this means that $D(u) u z D(u)=D(u) z u D(u)$ where $u \in U$ and $z \in R$ are arbitrary. Since $D(u)$ commutes with $u$ (Step 1 ), this relation can be rewritten as $D(u) u z D(u)=D(u) z D(u) u$; now apply Lemma 3. 0 .

STEP 4. (i) For $u, v \in U$, either $D(u)(v-\lambda(v))=0$ or $D(v)(u-\lambda(u))=$

(ii) For $u, v \in U$, either $(v-\lambda(v)) D(u)=0$ or $(u-\lambda(u)) D(v)=0$.

Proof. A linearization of $[D(u), u]=0, u \in U$, gives $[D(u), v]+$ $[D(v), u]=0, u, v \in U$. Replacing $v$ by $u v$ we get

$$
\begin{aligned}
0= & {[D(u), u v]+[u D(v)+D(u) v, u] } \\
= & {[D(u), u] v+u\{[D(u), v]+[D(v), u]\} } \\
& +D(u)[v, u]+[D(u), u] v .
\end{aligned}
$$

Note that in the last sum, every summand except possibly $D(u)[v, u]$ is 0 ; but then $D(u)[v, u]=0$ as well. That is, $D(u) v(u-\lambda(u))=0, u, v \in U$. By Steps 1, 2 and 3, $U$ contains elements of the form $(v-\lambda(v)) x D(v), v \in U$, $x \in R$. Therefore the last relation yields $D(u)(v-\lambda(v)) x D(v)(u-\lambda(u))=0$ for all $u, v \in U, x \in R$. Since $R$ is prime, this proves (i). In a similar fashion (first showing that $[v, u] D(u)=0$, and then applying Step 2) one proves (ii).

STEP 5. Either $D(U)=0$ or $U \subseteq Z$.

Proof. Take $v \in U$ and assume that $D(v) \neq 0$. Suppose that $D(u)(v-$ $\lambda(v)) \neq 0$ for some $u \in U$. Then $D(v)(u-\lambda(u))=0$ by Step 4. Now, consider the pair of elements $v$ and $u+v$. Since $D(u+v)(v-\lambda(v)) \neq 0$ (namely, $D(u)(v-\lambda(v)) \neq 0$ and $D(v)(v-\lambda(v))=0)$, it follows that $D(v)(u+v-\lambda(u+v))=0$. We have $D(v) u=\lambda(u) D(v), D(v) v=\lambda(v) D(v)$, so it follows that $D(v)(\lambda(u)+\lambda(v)-\lambda(u+v))=0$. By assumption, $D(v) \neq 0$, hence $\lambda(u+v)=\lambda(u)+\lambda(v)$. Consequently

$$
\begin{aligned}
0 & =D(u+v)((u+v)-\lambda(u+v)) \\
& =(D(u)+D(v))((u-\lambda(u))+(v-\lambda(v)) .
\end{aligned}
$$

Since $D(u)(u-\lambda(u))=0, D(v)(u-\lambda(u))=0$ and $D(v)(v-\lambda(v))=0$, it follows that $D(u)(v-\lambda(v))=0$, contrary to the assumption. Thus we 
have showed that if $D(v) \neq 0$ then $D(u)(v-\lambda(v))=0$ for all $u \in U$. Similarly one shows that in this case we also have $(v-\lambda(v)) D(u)=0$, $u \in U$. Combining both statements we see that $v$ commutes with all $D(u)$, $u \in U$. This means that $U$ is the union of its additive subgroups $G=\{v \in$ $U \mid D(v)=0\}$ and $H=\{v \in U \mid[D(u), v]=0$ for all $u \in U\}$. However, a group cannot be the union of two proper subgroups, hence either $G=U$, that is, $D(U)=0$, or $H=U$, that is, $D(U) \subseteq Z$ by the Corollary. In any case $D(U) \subseteq Z$. But then $D(u)(u-\lambda(u))=0$ implies $D(u) R(u-\lambda(u))=0$, and so for any $u \in U$ we have either $D(u)=0$ or $u \in C \cap R=Z$. Again using the fact that a group cannot be the union of two proper subgroups it follows that $D(U)=0$ or $U \subseteq Z$.

None of the assertions in Step 5 can hold-by Lemma 1, $D$ cannot vanish on $U$, and by Posner's theorem, $U$ cannot be contained in $Z$. The proof of the Theorem is thereby completed.

We conclude with an open question: is it possible to generalize the Theorem by proving that $U$ contains a nonzero two-sided ideal?

\section{References}

[1] H. E. Bell and W. S. Martindale, 'Semiderivations and commutativity in prime rings', Canad. Math. Bull. 31 (1988), 500-508.

[2] J. Bergen, I. N. Herstein, and J. W. Kerr, 'Lie ideals and derivations in prime rings', $J$. Algebra 71 (1981), 259-267.

[3] M. Brešar and J. Vukman, 'Jordan derivations on prime rings', Bull. Austral. Math. Soc. 37 (1988), 321-322.

[4] _ _ 'Orthogonal derivations and an extension of a theorem of Posner', Rad. Mat. 5 (1989), 237-246.

[5] $\ldots$, 'On left derivations and related mappings', Proc. Amer. Math. Soc. 110 (1990), $7-16$.

[6] I. N. Herstein, Topics in ring theory (Univ. of Chicago Press, Chicago, 1969).

[7] __ Rings with involution (Univ. of Chicago Press, Chicago, 1976).

[8] _., 'A note on derivations', Canad. Math. Bull. 21 (1978), 369-370.

[9] N. Jacobson, Structure of rings, Colloq. Publ. 37 (Amer. Math. Soc., Providence, RI, 1956).

[10] W. S. Martindale, 'Prime rings satisfying a generalized polynomial identity', J. Algebra 12 (1969), 576-584.

[11] E. C. Posner, 'Derivations in prime rings', Proc. Amer. Math. Soc. 8 (1957), 1093-1100.

[12] J. Vukman, 'Commuting and centralizing mappings in prime rings', Proc. Amer. Math. Soc. 109 (1990), 47-52.

University of Maribor

PF, Koroška

160, 62000 Maribor

Slovenia 